\title{
On the role of valence and semi-core electron correlation on spin-gaps in Fe(II)-Porphyrins
}

\author{
Giovanni Li Manni, ${ }^{*}$ Daniel Kats, ${ }^{*}$ David P. Tew, ${ }^{*}$ and Ali Alavi* \\ Max-Planck-Institut für Festkörperforschung, Heisenbergstraße 1, 70569 Stuttgart, \\ Germany \\ E-mail: g.limanni@fkf.mpg.de; d.kats@fkf.mpg.de; d.tew@fkf.mpg.de; a.alavi@fkf.mpg.de
}

\begin{abstract}
The role of valence and semi-core correlation in differentially stabilizing the intermediate spin-state of $\mathrm{Fe}(\mathrm{II})$-porphyrins is analyzed. CASSCF treatment of the valence correlation, with a $(32,34)$ active space containing metal $3 \mathrm{~d}$, $4 \mathrm{~d}$ orbitals and the entire $\pi$ system of the porphyrin, is necessary to stabilize the intermediate spin-state for this system. Semi-core correlation provides a quantitatively significant $(\sim 1.5 \mathrm{kcal} / \mathrm{mol})$ but less important correction. Accounting for both types of correlation enlarges the $\left({ }^{3} E_{g}-{ }^{5} A_{1 g}\right)$ spin-gap to $\sim-5 \mathrm{kcal} / \mathrm{mol}$.
\end{abstract}




\section{Introduction}

To date many questions have been left unanswered regarding the electronic structure and reactivity of metal-porphyrins. For instance, in spite of the large amount of experimental and theoretical data, a definitive understanding of the ground state electronic structure of the four-coordinated Fe(II)-porphyrins is still missing, and the electronic mechanisms ruling the relative stability of the spin-states along reaction pathways unknown.

In a recent work by Li Manni and Alavi, $\frac{1}{1}$ the stabilization of the triplet spin-state over the quintet spin-state in a Fe(II)-porphyrin model system has been explained on the basis of a complex interplay between ring correlation at the level of the $\pi$-system, a correlated breathing mechanism for the $3 \mathrm{~d}$ electrons of the metal center, and electron re-distribution between metal center and macrocycle via charge-transfer transitions. This has been made possible via explicit correlation of the valence electrons, using the novel Stochastic-CASSCF approach, ${ }^{2}$ with an active space containing 32 electrons in 34 orbitals. For the same model system, CASSCF calculations with active spaces up to CAS $(14,16)$, followed by second order perturbation theory correction, CASPT2, were not able to capture higher order correlation effects and orbital relaxation outside the active space, and as a consequence the high spin-state resulted over-stabilized with respect to the intermediate spin-state (see Figure 2 in Reference [ 1]). The CAS(32,34) is to be considered a valence only active space and the role of the semi-core correlation was not explicitly accounted for.

Another recent work by Phung, Pierloot and co-workers ${ }^{\sqrt{3}}$ develops around the well documented weakness of the CASPT2 approach, biased towards high spin-states due to a poor perturbational treatment of the transition metal semi-core (3s3p) shell. ${ }^{4}$ This problem is not solved using other zeroth-order Hamiltonians, such as the one used in the NEVPT2 approach. ${ }^{[-7}$ In their work, ${ }^{3}$ Pierloot and co-workers proposed a combined CASPT2/CC approach, with frozen semi-core CASPT2 in a large orbital basis, to recover large part of the valence correlation, and coupled cluster with singles, doubles, and perturbative triples correction, $\operatorname{CCSD}(\mathrm{T}), \frac{8}{10}$ in a smaller one-electron basis, to account for the $(3 \mathrm{~s} 3 \mathrm{p})$ semi-core correlation energy, $\Delta s p$. The message delivered by their work 
is that semi-core $(3 \mathrm{~s} 3 \mathrm{p})$ correlation contributes with a non-negligible $2.6 \mathrm{kcal} / \mathrm{mol}$ to the spin-gap estimate in favor of the intermediate spin-state. However, they also stated that "the original CASPT2 bias towards HS [high spin] states is not completely lifted in the $C A S P T 2 / C C^{\prime \prime}$, a clear indication that the failure of CASPT2 is not confined solely to semi-core correlation. It is also not able to fully capture valence correlation, even at a qualitative level, as already pointed out in Reference [ 1].

In this Letter, valence and semi-core correlation have been analyzed jointly, in an effort to understand and compare the role of the two forms of correlation, and to produce a unified mechanism that could explain spin-gaps in metal-porphyrins. Valence correlation effects, as captured by the $\operatorname{CASSCF}(32,34)$ method, are here discussed in greater detail, with focus on how total electron density is modified due to electron correlation (Section 3.1). The coupled cluster (CC) method is a reliable tool for describing spin-gaps in transition metal complexes, including Fe(II)-porphyrins, $\underline{3 \mid 1 / 1112}$ if static electron correlation is not playing a major role. In the present work, the coupled cluster method taken to high orders, and the distinguishable cluster method (DC), 13 support the Stochastic-CASSCF results. ${ }^{1}$ A strict comparison of the Stochastic-CASSCF and the $\mathrm{CC}$ methods in the same MO representation and same active space (AS-CC label) also provides a solid ground to discuss the accuracy in the truncation of the CC many-body expansion (here from CCSD to CCSDTQ) and possible sources of error in the Stochastic-CASSCF approach (Section 3.2). In order to capture additional correlation outside the $\operatorname{CAS}(32,34)$ three strategies have been undertaken. Our first strategy has been inspired by the approach proposed by Pierloot and co-workers, and consists in a combined CASSCF/CC method, with the Stochastic-CAS $(32,34)$ to recover the important valence correlation, overcoming the limitation of CASPT2, and the CC method for the semicore correlation (Section 3.5). The second strategy consists in larger CASSCF $(40,38)$ calculations, with the (3s3p) semi-core orbitals added to the $(32,34)$ active space, and correlated at the CASSCF level of theory (Section 3.6). Both methods provide an enlarged $\left({ }^{3} E_{g}-{ }^{5} A_{1 g}\right)$ spin-gap of $\sim 5 \mathrm{kcal} / \mathrm{mol}$, which is about $2 \mathrm{kcal} / \mathrm{mol}$ larger than the spin-gap predicted by the previous Stochastic-CASSCF $(32,34)$ approach, in line with 
the estimate suggested by Pierloot via the CASPT2/CC approach. Our third strategy consists of CCSD(T) and DCSD calculations in the full orbital expansion, FS-CC label (Section 3.3).

This investigation provides the means to better understand the role of valence and semi-core correlation in the differential stabilization of the intermediate spin-state over the high spin-state in metal-porphyrins. Valence correlation, as captured by the StochasticCASSCF $(32,34)$ approach, has an important qualitative effect. It massively stabilizes the triplet over the quintet and accounts for re-distribution of charges between the metal center and the macrocycle. Ring-correlation, radial-correlation and charge-redistribution are not captured by smaller active space calculations, thus leading to incorrect spin ordering. Semi-core correlation is a smaller, quantitative correction, yet crucial when considering the small spin gaps in metal-porphyrins.

\section{Computational Details}

Basis set choice, integrals evaluation, MCSCF procedure and setup of the QMC dynamics are unchanged compared to Reference [ 1] and will not be further discussed here. The $(32,34)$ active space, chosen in our earlier work ${ }^{11}$ consists of the four doubly occupied $\sigma$ orbitals on the macrocycle, pointing at the metal center, the entire $\pi$ system (18 electrons in 16 orbitals), five 3d orbitals and their six electrons, five empty correlating d' orbitals, and the empty $(4 \mathrm{~s} 4 \mathrm{p})$ shell.

The structure of the model system utilized in the present investigation is identical to the one used in Reference [ 1]. In this model, the carbon atoms in $\beta$-positions have been replaced with $\mathrm{H}$ atoms. An active space for the expanded Fe(II)-porphyrin, equivalent to the one here utilized, consists of 40 electrons in 42 orbitals, CAS $(40,42)$, that is enlarged by the eight electrons and the eight $\pi$ orbitals of the $\beta$-carbon atoms, and will be presented elsewhere. The choice of the smaller model system has been made to simplify the computations, yet all chemical properties of the metal-porphyrin have been preserved, including the conjugation at the level of the macro-cycle, in our view crucial 
to describe ring-correlation and to make a connection between the frontier orbitals of the $\pi$ system and Gouterman's orbitals. The geometry of this model system is based on the triplet geometry of the Fe(II)-porphyrin (with a small Fe-N bond length). This certainly represents a bias towards the intermediate spin-state that needs to be addressed. Still, we find it a useful model system, especially considering that even in this biased structure the CASPT2 approach provides a qualitatively wrong spin gap estimate.

The inclusion of the four semi-core orbitals and their eight electrons in the $(32,34)$ active space leads to a $(40,38)$ active space. For this larger active space in the FCIQMC dynamic the population was increased to up to 4 billion walkers.

For a close comparison with the $\operatorname{CASSCF}(32,34)$ and $\operatorname{CASSCF}(40,38)$ energetics, CC and DC calculations have been performed inside the $(32,34)$ and the $(40,38)$ active spaces (AS-CC label) from the CASSCF calculations. CC calculations in the full orbital expansion (FS-CC label) have also been performed to capture additional correlation outside the active space. For these calculations two one-electron basis have been used, the canonical ROHF orbitals and the CAS $(32,34)$ natural orbitals.

All the CASSCF, Stochastic-CASSCF and CASPT2 calculations were performed using the OpenMolcas chemistry software package, $\frac{16}{16}$ while the CC and DC calculations, were performed using the MOLPRO ${ }^{17}$ and the MRCC ${ }^{18}$ software packages. An ad hoc interface was created to translate orbital coefficients from the Molcas to the MOLPRO format.19

\section{Results}

\subsection{Valence correlation via Stochastic-CASSCF $(32,34)$}

In our earlier investigation ${ }^{1}$ a correlation mechanism was provided that contains two important elements, leading to the differential stabilization of the intermediate spinstate over the high spin-state. Ring correlation is the first of these elements and it is responsible for a reduced electron repulsion among the $\pi$ electrons, effectively making of the macrocycle a better electron acceptor. The correlated $\pi$ natural orbitals, obtained from the Stochastic-CASSCF $(32,34)$, and in particular the four Gouterman's orbitals, 
have occupation numbers that substantially deviate from two and zero (as shown in Figure 5 of Reference [ 1]), indicating the presence of important correlation at the level of the macrocycle. This correlation would be missed in smaller active space calculations. The fact that CASSCF $(32,34)$ differentiates Gouterman's orbitals from the rest of the $\pi$ system is striking, for it represents an important ab initio result in support of Gouterman's model, suggested more than 50 years ago on the basis of symmetry arguments. $\frac{20121}{2}$

The double-shell, $d^{\prime}$, orbitals are the second key element of the proposed mechanism. They play a dual role, as they provide necessary flexibility for radial correlation and orbital relaxation (correlated breathing), and maximize the overlap with the $\pi$ system, facilitating charge-transfer processes between metal and ligand. Together these factors are responsible for a re-distribution of charges between metal and macrocycle that is energetically favorable for the intermediate spin-states.

The re-distribution of charges due to electron correlation are shown here in a simple and compact manner, by analyzing total electron densities in real-space for the correlated CAS $(32,34)$ and the uncorrelated ROHF wave functions. The difference of the correlated CAS $(32,34)$ and uncorrelated ROHF total electron density for the triplet and the quintet spin-states is shown in Figure1. The green areas represent regions with reduced electron
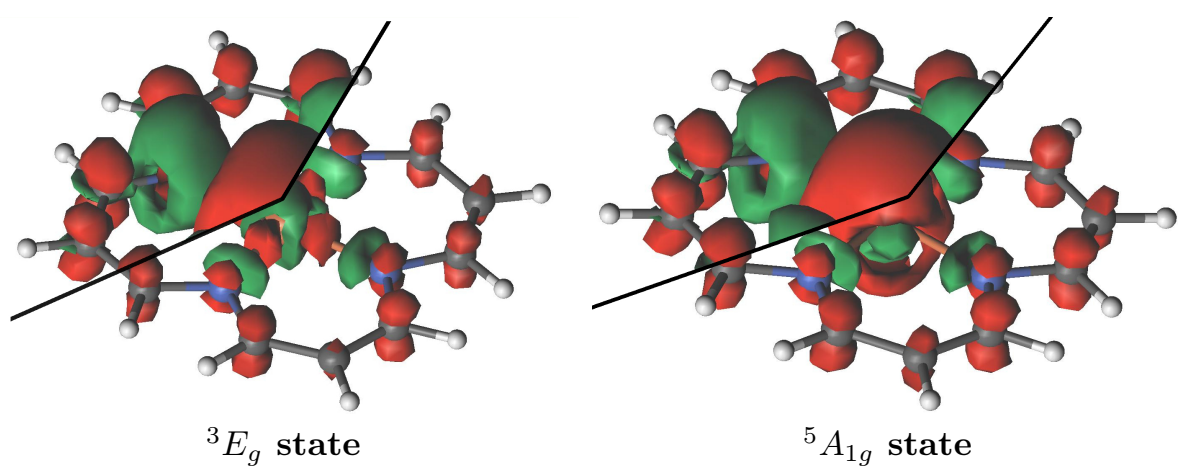

Figure 1: Real space total electron density difference between correlated StochasticCASSCF $(32,34)$ and ROHF wave functions for the ${ }^{3} E_{g}$ (left) and the ${ }^{5} A_{1 g}$ (right) states. For the upper-left and the lower-right corners values of 0.01 and 0.03 were used for the iso-surface contour level, respectively.

population due to correlation, whilst red areas represent regions with increased electron density. For both spin-states there is a similar re-distribution of electron density upon correlation. Electron density is reduced in the regions of the nitrogen lone-pairs and 
at the level of the $3 \mathrm{~d}$ orbitals in the metal center, and increased in the outer region of the metal center, mostly with spherical distribution (4s orbital) and at the level of the $\pi$-system. Considering that in the ${ }^{3} E_{g}$ state the $d_{x^{2}-y^{2}}$ orbital (the one on the plane and not pointing at the nitrogen atoms) is formally doubly occupied, it is not surprising that the green region at the metal center has the symmetry of this orbital. Analogously for the quintet spin-state the green region has the shape of the formally doubly occupied $d_{z^{2}}$ orbital. These charge redistributions are a consequence of the mechanism discussed earlier, ${ }^{1}$ with radial-correlation and breathing effects allowing $3 \mathrm{~d}$ electrons to expand out, and a correlated $\pi$-system with enhanced electron acceptor features. This mechanism can be simply described as a correlation enhanced $\sigma$-donation $/ \pi$-back-donation. This mechanism differentially reduces the on-site electron repulsion and energetically favors the triplet spin-state. The same mechanism can be invoked for the observed strengthening of the metal-ligand bond length for the intermediate spin-state.

In Figure 2 the difference of the correlated $\operatorname{CAS}(8,11)$ and uncorrelated ROHF total electron density is also given for comparison. The $\mathrm{CAS}(8,11)$ active space includes only
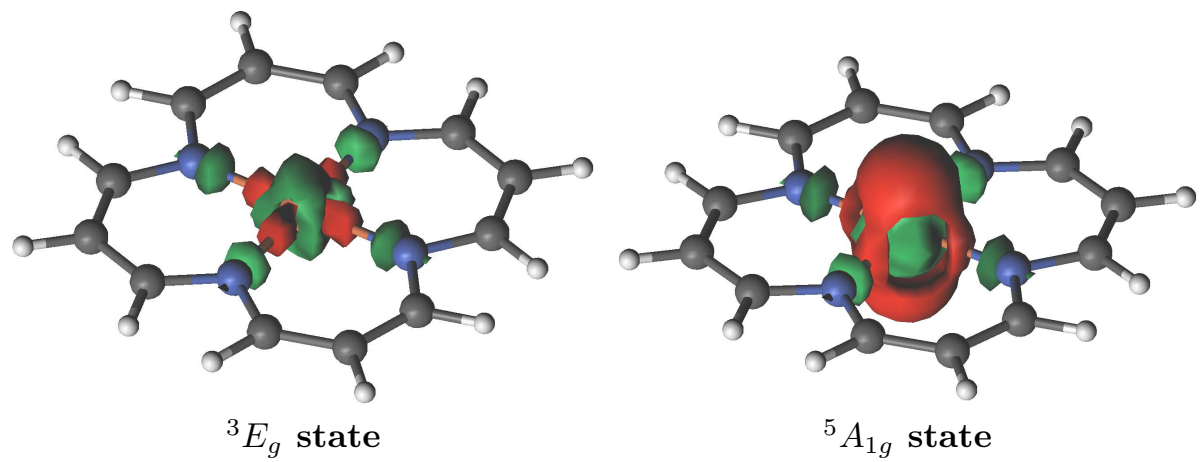

Figure 2: Real space total electron density difference between correlated $\operatorname{CASSCF}(8,11)$ and ROHF wave functions for the ${ }^{3} E_{g}$ (left) and the ${ }^{5} A_{1 g}$ (right) states.

the five $3 d$, the five $d^{\prime}$ and the bonding $\sigma$-orbital. This active space captures only part of the $\sigma$-donation and radial correlation effect at the metal center. Re-distribution of density at the macrocycle is not observed. Correlation of the $\pi$-electrons is not accounted for even indirectly via orbital mixing. Metal-only active spaces are incapable to capture charge re-distribution, which are only partially captured by subsequent PT2 treatments. 
The following quantity

$$
\left(\rho\left[{ }^{3} E_{g}(32,34)\right](r)-\rho\left[{ }^{3} E_{g}(R O H F)\right](r)\right)-\left(\rho\left[{ }^{5} A_{1 g}(32,34)\right](r)-\rho\left[{ }^{5} A_{1 g}(R O H F)\right](r)\right)
$$

shows for which spin-state there is a larger charge re-distribution due to correlation, and is reported in Figure 3. Figure 3 indicates that the ligand-to-metal $\sigma$-donation and

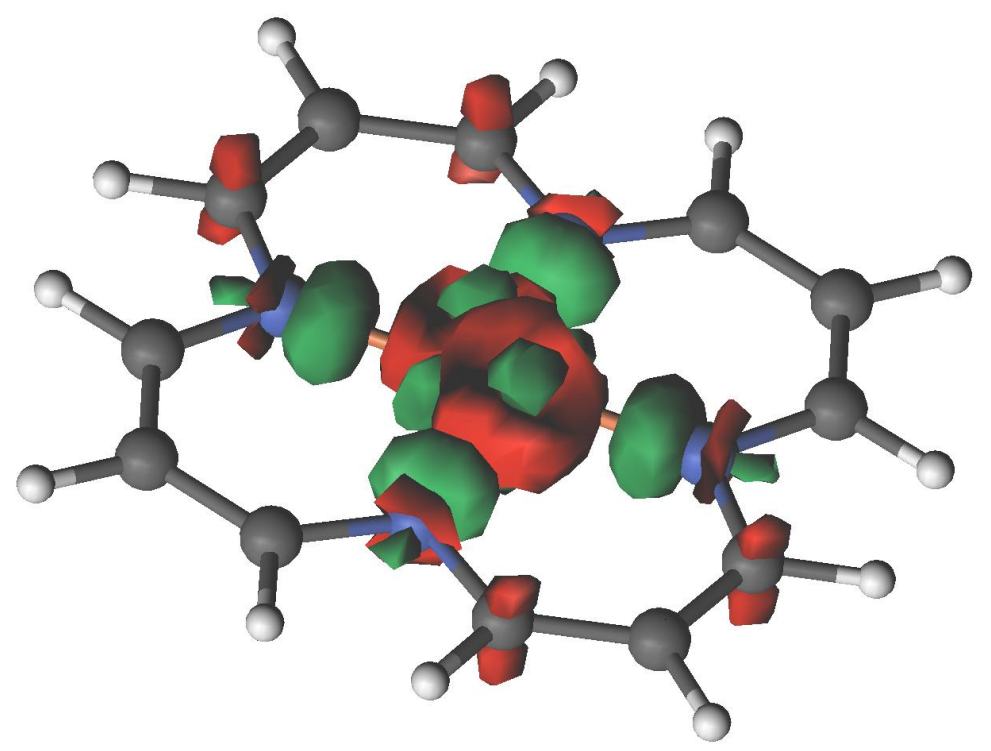

Figure 3: Density difference as given by Equation 1. Positive and negative differences are in red and green, respectively.

the metal-to-ligand $\pi$-back-donation are stronger in the triplet spin-state, confirming that the $\sigma$-donation $/ \pi$-back-donation mechanism energetically favors the triplet spinstate. Differences at the metal center are related to the different character of the radial correlation at the level of the $3 d$ orbitals.

\subsection{Valence correlation via the coupled cluster approach}

Active space only CC calculations (AS-CC label) have been performed, using the optimized CASSCF $(32,34)$ orbitals from our previous work, ${ }^{1}$ for a rigorous comparison between the Stochastic-CASSCF $(32,34)$ and the CC energy estimates. In this approach, the CAS(32,34) inactive and virtual orbitals were kept frozen, while an ROHF orbital relaxation was performed within the active orbitals. The most significant AS-CC results are summarized in Table 1. The CCSD method is clearly lacking an important part of 
Table 1: ${ }^{3} E_{g}$ and ${ }^{5} A_{1 g}$ absolute energies (a.u.) and spin-gap (kcal/mol) within the $(32,34)$ active space for various truncation of the many-body expansion in the CC ansatz.

\begin{tabular}{ccccccc}
\hline State & CAS $(32,34)$ & CCSD & DCSD & CCSD(T) & CCSDT & CCSDTQ \\
\hline${ }^{3} E_{g}$ & -1951.3580 & -1951.3366 & -1951.3508 & -1951.3538 & -1951.3580 & -1951.3596 \\
${ }^{5} A_{1 g}$ & -1951.3530 & -1951.3353 & -1951.3466 & -1951.3495 & -1951.3530 & -1951.3539 \\
$\Delta E$ & -3.1 & -0.8 & -2.6 & -2.7 & -3.1 & -3.6 \\
\hline
\end{tabular}

correlation, predicting a small spin-gap. $\operatorname{CCSD}(\mathrm{T})$ and DCSD predictions improve over CCSD with a $\left({ }^{3} E_{g}-{ }^{5} A_{1 g}\right)$ spin-gap close to the Stochastic-CAS $(32,34)$ result, a satisfactory result, but still slightly underestimating the spin-gap. CCSDT provides energetics that closely agree with the Stochastic-CAS $(32,34)$, whereas the CCSDTQ spin-gap estimate is $0.5 \mathrm{kcal} / \mathrm{mol}$ larger than the Stochastic-CASSCF $(32,34)$ estimate. This latter result indicates that the Stochastic-CASSCF $(32,34)$, although very satisfactory, is not entirely converged with respect to the walker population, enlarged up to 1 million walkers in our previous report. 1 The initiator approximation employed in the FCIQMC calculations ${ }^{22}$ could be responsible for size-consistency issues and undersampling of higher order excitations. Table 1 also indicates that higher order many-body terms play an important differential role in spin-gap predictions for metal-porphyrins, and even at the CCSD $(\mathrm{T})$ level of theory, the spin-gap is underestimated by $\sim 1 \mathrm{kcal} / \mathrm{mol}$, when compared to the CCSDTQ counterpart.

\subsection{Full space coupled cluster approach}

Coupled cluster spin-gap estimates on the entire orbital expansion (FS-CC label) are collected in Table 2. Two one-electron basis have been used, the canonical ROHF orbital set and the $\operatorname{CAS}(32,34)$ natural orbital expansion. The role of the semi-core correlation has been investigated in both basis, by performing calculations where the semi-core electrons have been excluded $\left(\Delta E_{\text {nosp }}\right)$ or included $\left(\Delta E_{+s p}\right)$ in the CC treatment. Considering the size of the basis set (707 contracted basis functions), only CCSD, DCSD and CCSD(T) have been employed, higher-order many-body expansions being prohibitively expensive. The results shown in Table 2 show that CCSD provides qualitatively erratic spin-gap estimates, independently of the MO basis used, with the triplet spin state at $2.3 \mathrm{kcal} / \mathrm{mol}$ 
Table 2: $\left({ }^{3} E_{g}-{ }^{5} A_{1 g}\right)$ spin-gap (kcal/mol) on the basis of canonical ROHF and CAS $(32,34)$ natural orbitals. The $T_{1}^{s p}$ column corresponds to calculations with semi-core excitations restricted to singles.

\begin{tabular}{cccccccc}
\hline State & \multicolumn{2}{c}{ CCSD } & \multicolumn{2}{c}{ CCSD(T) } & \multicolumn{2}{c}{ DCSD } & CASPT2(8,11) \\
& ROHF & CAS $(32,34)$ & ROHF & CAS $(32,34)$ & ROHF & $T_{1}^{s p}$ & \\
\hline$\Delta E_{n o s p}$ & +4.0 & +5.0 & -0.4 & -1.5 & +1.8 & +0.2 & +1.6 \\
$\Delta E_{+s p}$ & +2.3 & +4.0 & -2.7 & -3.2 & -0.5 & -0.5 & +2.9 \\
$\Delta_{s p}$ & -1.7 & -1.0 & -2.3 & -1.7 & -2.3 & -0.7 & +1.3 \\
\hline
\end{tabular}

above the ${ }^{5} A_{1 g}$ state $(4.0 \mathrm{kcal} / \mathrm{mol}$ in the $\operatorname{CAS}(32,34)$ natural MO basis). This result is quite unexpected, considering that in the previous AC-CC calculations CCSD was qualitatively correct. At the $\operatorname{CCSD}(\mathrm{T})$ level of theory, correlation of the semi-core electrons enlarges the spin-gap (in favor of the intermediate spin-state) by $2.3 \mathrm{kcal} / \mathrm{mol}$ and $1.7 \mathrm{kcal} / \mathrm{mol}$, in the canonical ROHF and the CAS $(32,34)$ natural orbital basis, respectively. In Table 3 of Reference [ 3] a $\Delta_{s p}$ of $-2.61 \mathrm{kcal} / \mathrm{mol}$ was reported for the FeP model system, in good agreement with the findings here reported. Using the CAS $(32,34)$ natural orbitals as basis for the frozen semi-core CC calculations has the effect of enlarging the gap by $\sim 1 \mathrm{kcal} / \mathrm{mol}$ in favor of the triplet, when compared to the gap obtained on the basis of the ROHF orbitals. The orbital relaxation of the (3s3p) shell in the preceding CASSCF $(32,34)$ is to be considered responsible for this effect, as discussed in greater detail in Section 3.4. The spin-gap from DCSD is smaller than in the active space only calculations, but the effect of the semi-core is the same as for $\operatorname{CCSD}(\mathrm{T})$.

In the development version of MOLPRO an option has been implemented, to allow only single excitations from selected orbitals, in order to identify orbital relaxation effects and distinguish them from other forms of electron correlation. Allowing single excitations from the semi-core orbitals at the DCSD level lowers the triplet state by $1.6 \mathrm{kcal} / \mathrm{mol}$ (column $T_{1}^{s p}$ in Table 2) accounting for $70 \%$ of the semi-core effect, the remaining 0.7 $\mathrm{kcal} / \mathrm{mol}$ being related to other forms of correlation.

\subsection{How semi-core orbitals are modified by valence correlation}

In this section the indirect effect of valence correlation, due to the variational orbital optimization, onto the semi-core shell is analyzed. The ROHF $3 s$ orbital of the triplet 
spin-state is surprisingly similar to the ROHF $3 s$ orbital of the high spin-state (upper-left orbital of Figure 4). In the $\operatorname{CASSCF}(32,34)$ approach, the $3 s$ orbital of both spin-states mix with the $\sigma$-orbitals of the ligand (upper-right orbital of Figure 4). This mixing is, however, different for the two spin-states, and as a consequence the $3 s$ orbital is different in the two states (see bottom plot of Figure 4). These findings suggest that there is
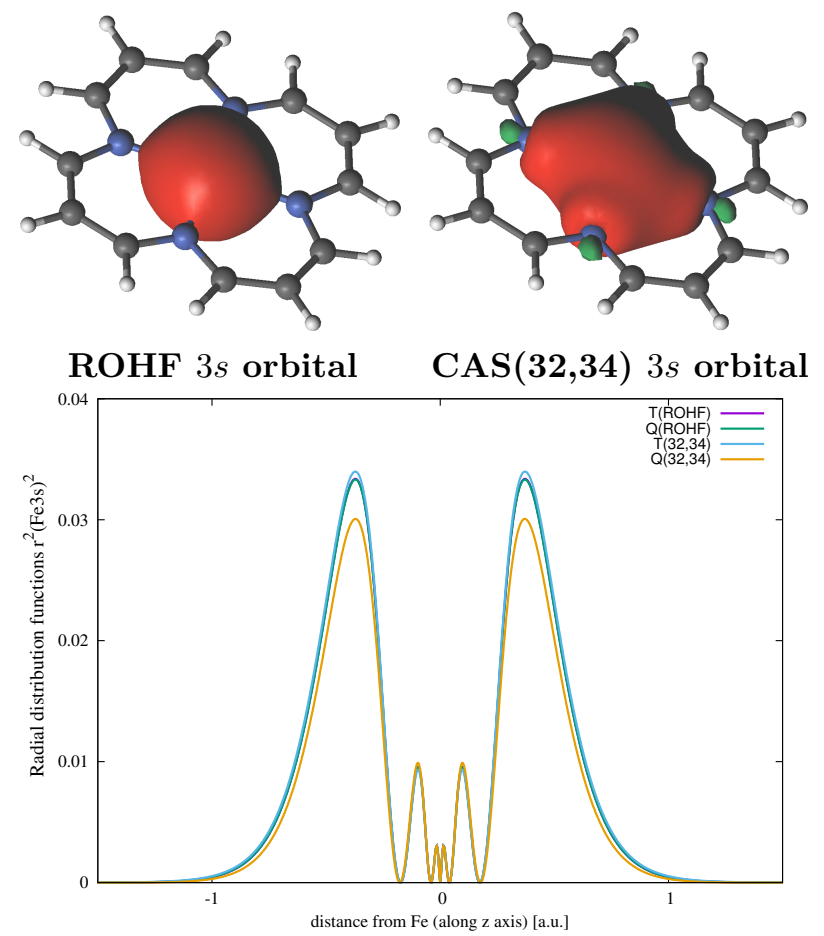

Radial distribution function

Figure 4: ROHF (left) and $\operatorname{CAS}(32,34)$ (right) $3 s$ orbital for the ${ }^{3} E_{g}$ state. Radial distribution function of the 3s orbital (along the axis perpendicular to the molecular plane) for the two spin-states in the correlated CAS $(32,34)$ and the ROHF wave functions (bottom).

a certain amount of (semi-)core/valence correlation that contributes to the differential stabilization of the intermediate spin-state over the high spin-state. This core-valence correlation is partially accounted for via orbital relaxation at the $\mathrm{CASSCF}(32,34)$ level, whereas it is missing in smaller active space calculations. The results obtained via FC$\mathrm{CC}$ can be explained in light of this core-valence correlation. When the semi-core are not correlated at the CC level of theory, and using the ROHF basis, any form of corevalence correlation is missing, leading to a substantial error in the spin-gap estimates. The spin-gap predictions are improved when the semi-core orbitals are correlated and core-valence correlation accounted for. When using the $\operatorname{CASSCF}(32,34)$ natural orbitals 
as basis for CC calculations, part of the core-valence correlation is recovered via semicore/valence orbital mixing, already at the CASSCF level. This explains the improvement of $\sim 1 \mathrm{kcal} / \mathrm{mol}$ in the spin-gap estimates for the frozen semi-core CC calculations. A similar improvement is captured by the $T_{1}^{s p}$ approach reported in Table 2 . When the semicore shell is fully correlated at the CC level and in the CAS(32,34) MO basis, a larger portion of the core-valence correlation is recovered (higher order excitations) leading to further improved results. The same argument could be used to explain the failure of the CASPT2. Given the differential role of the core-valence correlation for the Fe(II)porphyrin system, it is legitimate to look for methods that explicitly account for this form of correlation. The method suggested in Reference [ 3] is a valid one, although not sufficient for the valence correlation. In the following two sections two other methods will be discussed to tackle core-valence correlation.

\subsection{Semi-core correlation via a combined $\operatorname{CASSCF}(32,34) / \mathrm{CC}$ approach}

Inspired by the work reported in Reference [ 3], we applied the CASPT2/CC approach to the small model system here investigated. A small $(8,11)$ active space was chosen, that includes five $3 d$ orbitals with their six electrons, five correlating $d^{\prime}$ orbitals and one doubly occupied metal-ligand $\sigma$-bonding orbital. CASPT2 $(8,11)$ calculations where performed for the ${ }^{3} E_{g}$ and the ${ }^{5} A_{1 g}$ states. CASPT2 $(8,11)$ produced qualitatively erroneous spin ordering (last column in Table 22). It is observed that when semi-core electrons are correlated at the CASPT2 level, an unfavorable effect on the spin-gap is obtained, with the quintet being stabilized more than the triplet spin-state, and a spin-gap of $+2.9 \mathrm{kcal} / \mathrm{mol}$. This aspect has been also reported earlier. ${ }^{3}$

If the $\operatorname{CCSD}(\mathrm{T}) \Delta_{s p}$ on the ROHF basis $(-2.3 \mathrm{kcal} / \mathrm{mol})$ is added to the frozen semicore CASPT2 $(8,11)$ spin-gap, $\Delta E_{n o s p}=+1.6 \mathrm{kcal} / \mathrm{mol}$, a small CASPT2 $/ \mathrm{CC}$ spin-gap is obtained $(-0.7 \mathrm{kcal} / \mathrm{mol})$. A similar small value is reported in Reference [ 3], leading to the conclusion that the high spin-state bias of the CASPT2 is not completely lifted by the CASPT2/CC approach. 
A different, and more controlled, option is to add the $\operatorname{CCSD}(\mathrm{T}) \Delta_{s p}$, in the basis of the $\operatorname{CAS}(32,34)$ natural orbitals $(-1.7 \mathrm{kcal} / \mathrm{mol})$, to the $\operatorname{CASSCF}(32,34)$ spin-gap in the same basis. In this case the same basis is used for the CASSCF and the CC semi-core correction, and a substantially larger spin-gap is obtained $(-4.8 \mathrm{kcal} / \mathrm{mol})$. This result, however, is not in agreement with the $\operatorname{CCSD}(\mathrm{T})$ prediction in the same basis (with a spin-gap of only $-3.2 \mathrm{kcal} / \mathrm{mol}$ ). This discrepancy can be explained considering either that the absence of higher order excitations in the $\operatorname{CCSD}(\mathrm{T})$ leads to a higher energy for the triplet spin-state, or that in the combined $\mathrm{CASSCF}(32,34) / \mathrm{CC}$ approach an artificial over-stabilization of the triplet spin-state emerges.

\subsection{Adding semi-core orbitals to the active space}

A more costly, but effective way to analyze the role of the semi-core orbitals is to include them explicitly in the active space, for an even larger CASSCF $(40,38)$ calculation. Considering that the $(4 \mathrm{~s} 4 \mathrm{p})$ shell is already in the $\operatorname{CAS}(32,34)$ discussed earlier, the addition of the (3s3p) shell would allow the two shells to couple explicitly providing the necessary flexibility for these orbitals to undergo correlated breathing (via radial correlation) in addition to other forms of electron correlation. At this level of theory the $\left({ }^{3} E_{g}-{ }^{5} A_{1 g}\right)$ spin-gap is $-4.4 \mathrm{kcal} / \mathrm{mol}$ (Table 3), noticeably larger than the spin-gap predicted at CASSCF $(32,34)$ level. This result is in good agreement with the CASSCF/CC results

Table 3: ${ }^{3} E_{g}$ and ${ }^{5} A_{1 g}$ energies (a.u.) and spin-gap (kcal/mol) within the $(40,38)$ active space.

\begin{tabular}{ccccccc}
\hline State & CAS $(40,38) / 4 \mathrm{~B}$ & CCSD & DCSD & CCSD $(T)$ & CCSDT & CCSDTQ \\
\hline${ }^{3} E_{g}$ & -1951.4360 & -1951.4142 & -1951.4281 & -1951.4315 & -1951.4357 & -1951.4372 \\
${ }^{5} A_{1 g}$ & -1951.4290 & -1951.4124 & -1951.4232 & -1951.4253 & -1951.4286 & -1951.4295 \\
$\Delta E$ & -4.4 & -1.2 & -3.1 & -3.9 & -4.4 & -4.8 \\
\hline
\end{tabular}

reported in the previous section. Coupled cluster results in the optimized $(40,38)$ active space $(\mathrm{AC}-\mathrm{CC})$ are also reported in Table 3 . Spin-gaps of $-3.9 \mathrm{kcal} / \mathrm{mol},-4.4 \mathrm{kcal} /-$ mol and $-4.8 \mathrm{kcal} / \mathrm{mol}$ are obtained for $\operatorname{CCSD}(\mathrm{T})$, CCSDT and CCSDTQ, respectively. Although the Stochastic-CASSCF with 4 billion (4B) walkers provides absolute energies that are lower than the CCSDT, their spin-gap predictions are comparable, setting the 
Stochastic-CASSCF in between the CCSD(T) and the CCSDTQ methods in terms of accuracy. Same as for the $\operatorname{CAS}(32,34)$ this result can be improved by further increasing the walker population. However, the result is satisfactory and further improvement not required to draw the relevant conclusions. The difference between the $\operatorname{CCSD}(\mathrm{T})$ and the CCSDTQ spin-gap indicates that part of the higher order many-body correlation is missing in the former method. This error is also contained in the $\operatorname{CCSD}(\mathrm{T})$ computations for the entire orbital space and could be used to justify why the FS-CCSD(T) spin-gap predictions are smaller than the AC-CC counterpart. Considering the agreement between the CASSCF (40,38), the AC-CC(40,38), and the $\operatorname{CASSCF}(32,34) / \mathrm{CC}$ methods here reported, $\sim-5 \mathrm{kcal} / \mathrm{mol}$ is our most accurate estimate for the triplet-quintet spin-gap for the model system here investigated.

\section{Conclusions}

By means of Stochastic-CASSCF, coupled cluster, and their combination (CASSCF/CC), the role of valence and semi-core correlation in the relative energy ordering of the high and the intermediate spin-states in a model system of the Fe(II)-porphyrin has been investigated in great detail. Valence correlation, as captured by $\operatorname{CASSCF}(32,34)$ has a massive effect on the relative energy of the spin-states. The $\left({ }^{3} E_{g}-{ }^{5} A_{1 g}\right)$ spin-gap obtained at $\operatorname{CASSCF}(32,34)$ level is $13 \mathrm{kcal} / \mathrm{mol}$ and $6 \mathrm{kcal} / \mathrm{mol}$ smaller than the one predicted by CASSCF $(8,12)$ and CASPT2 $(8,12)$, respectively (see Figure 2 of Reference ${ }^{1}$ ). Valence correlation has also a noticeable qualitative effect on the structure of the wave function, largely modified with respect to less correlated wave functions. Via the large StochasticCASSCF $(32,34)$ calculations, the Gouterman's model and a re-distribution of charge between the metal center and the macrocycle have been demonstrated. The charge redistribution can be explained by a complex mechanism that involves ring-correlation at the macrocycle, radial-correlation at the metal center (breathing effect") and chargetransfer excitations. This mechanism can be related to the well known $\sigma$-donation / $\pi$-back-donation process, that is manifested in the correlated wave function here used, 
otherwise quenched when more constrained wave functions are built, such as in the smaller $\mathrm{CAS}(8,12)$ or the restricted active space cases. The mechanism is differential and stronger for the triplet, that, as a consequence, is stabilized more than the high spin-state, leading to an inversion of energy order between the states.

At the $\operatorname{CASSCF}(32,34)$ level of theory also the semi-core orbitals are different in shape for the two spin-estimates. This effect is not present in smaller active space or ROHF wave functions, for which no differences are found for the (3s $3 p)$ orbitals between the two spin-states. This effect is driven by the orbital relaxation occurring during the variational optimization of the $\operatorname{CASSCF}(32,34)$ wave function. The single excitations from the semi-core orbitals at the DCSD level also indicate that a large portion of the semi-core correlation can be attributed to orbital relaxation. When the (3s $3 p)$ shell is explicitly correlated, and higher order excitations included, the intermediate spinstate is stabilized even more with respect to the quintet state. This is observed at the CC and DC level, at the CASPT2/CC level,,$\frac{3}{\text { via }} \operatorname{CASSCF}(32,24) / \mathrm{CC}$ and in the CASSCF $(40,38)$, and is related to the semi-core/valence correlation. Explicit evaluation of semi-core correlation together with a CCSDTQ correction to the valence correlation leads to an improved spin-gap prediction of about $-5 \mathrm{kcal} / \mathrm{mol}$ for the model system here presented, a non negligible improvement if compared to the $\sim-3 \mathrm{kcal} / \mathrm{mol}$ spin-gap obtained in our previous work. 1

Thus, from our investigation, two forms of correlation can be distinguished, valence and semi-core correlation. The former has a massive effect on the spin gap estimates and is qualitative in character, for it qualitatively changes the structure of the wave function and is responsible for the charge re-distribution and the enhanced $\sigma$-donation $/ \pi$-backdonation process. Semi-core correlation represents a very important quantitative effect, for spin-gap predictions for ferrous-porphyrins.

\section{Competing Financial Interests statement}

The authors declare no competing financial interest. 


\section{Correspondence}

Correspondence and requests for materials should be addressed to g. limanni@fkf .mpg . de, d.kats@fkf.mpg.de, and a.alavi@fkf.mpg.de.

\section{References}

(1) Li Manni, G.; Alavi, A. J. Phys. Chem. A 2018,

(2) Li Manni, G.; Smart, S. D.; Alavi, A. J. Chem. Theory Comput. 2016, 12, 12451258.

(3) Phung, Q. M.; Feldt, M.; Harvey, J. N.; Pierloot, K. J. Chem. Theory Comput. 2018, 14, 2446-2455.

(4) Pierloot, K.; Phung, Q. M.; Domingo, A. J. Chem. Theory Comput. 2017, 13, $537-553$.

(5) Angeli, C.; Cimiraglia, R.; Evangelisti, S.; Leininger, T.; Malrieu, J.-P. J. Chem. Phys. 2001, 114, 10252-10264.

(6) Angeli, C.; Cimiraglia, R.; Malrieu, J.-P. J. Chem. Phys. 2002, 117, 9138-9153.

(7) Angeli, C.; Borini, S.; Cavallini, A.; Cestari, M.; Cimiraglia, R.; Ferrighi, L.; Sparta, M. Int. J. Quantum Chem. 2006, 106, 686-691.

(8) Čížek, J. J. Chem. Phys. 1966, 45, 4256.

(9) Purvis, G. D.; Bartlett, R. J. J. Chem. Phys. 1982, 76, 1910.

(10) Raghavachari, K.; Trucks, G. W.; Pople, J. A.; Head-Gordon, M. Chem. Phys. Lett. 1989, 15\%, 479 .

(11) Radoń, M. J. Chem. Theory Comput. 2014, 10, 2306-2321.

(12) Chen, H.; Lai, W.; Shaik, S. J. Phys. Chem. Lett. 2010, 1, 1533-1540. 
(13) Kats, D.; Manby, F. R. J. Chem. Phys. 2013, 139, 021102.

(14) Kats, D. J. Chem. Phys. 2014, 141, 061101.

(15) Kats, D. J. Chem. Phys. 2016, 144, 044102.

(16) Aquilante, F.; Autschbach, J.; Carlson, R. K.; Chibotaru, L. F.; Delcey, M. G.; De Vico, L.; Fdez. Galván, I.; Ferré, N.; Frutos, L. M.; Gagliardi, L.; Garavelli, M.; Giussani, A.; Hoyer, C. E.; Li Manni, G.; Lischka, H.; Ma, D.; Malmqvist, P.Å.; Müller, T.; Nenov, A.; Olivucci, M.; Pedersen, T. B.; Peng, D.; Plasser, F.; Pritchard, B.; Reiher, M.; Rivalta, I.; Schapiro, I.; Segarra-Martí, J.; Stenrup, M.; Truhlar, D. G.; Ungur, L.; Valentini, A.; Vancoillie, S.; Veryazov, V.; Vysotskiy, V. P.; Weingart, O.; Zapata, F.; Lindh, R. J. Comput. Chem. 2016, 37, $506-541$.

(17) Werner, H.-J.; Knowles, P. J.; Knizia, G.; Manby, F. R.; Schütz, M.; Celani, P.; Györffy, W.; Kats, D.; Korona, T.; Lindh, R.; Mitrushenkov, A.; Rauhut, G.; Shamasundar, K. R.; Adler, T. B.; Amos, R. D.; Bernhardsson, A.; Berning, A.; Cooper, D. L.; Deegan, M. J. O.; Dobbyn, A. J.; Eckert, F.; Goll, E.; Hampel, C.; Hesselmann, A.; Hetzer, G.; Hrenar, T.; Jansen, G.; Köppl, C.; Liu, Y.; Lloyd, A. W.; Mata, R. A.; May, A. J.; McNicholas, S. J.; Meyer, W.; Mura, M. E.; Nicklass, A.; O’Neill, D. P.; Palmieri, P.; Peng, D.; Pflüger, K.; Pitzer, R.; Reiher, M.; Shiozaki, T.; Stoll, H.; Stone, A. J.; Tarroni, R.; Thorsteinsson, T.; Wang, M. MOLPRO, version 2015.1, a package of ab initio programs. 2015; see http://www.molpro.net.

(18) Kállay, M.; Rolik, Z.; Csontos, J.; Ladjánszki, I.; Szegedy, L.; Ladóczki, B.; Samu, G. MRCC, a Quantum Chemical Program Suite. see also Z. Rolik, L. Szegedy, I. Ladjánszki, B. Ladóczki, and M. Kállay, J. Chem. Phys. 139, 094105 (2013), as well as: www.mrcc.hu.

(19) Mol-Cas-Pro, scripts to transform Molcas orbital coefficients to MOLPRO. see https://bitbucket.org/kats/molcaspro. 
(20) Gouterman, M. J. Chem. Phys. 1959, 30, 1139-1161.

(21) Gouterman, M. J. Mol. Spectrosc. 1961, 6, 138-163.

(22) Cleland, D.; Booth, G. H.; Alavi, A. J. Chem. Phys. 2010, 132, 041103. 